\title{
Pulse Compression of Phase-matched High Harmonic Pulses from a Time-Delay Compensated Monochromator
}

\author{
Hironori Igarashi, Ayumu Makida, Motohiko Ito, and Taro Sekikawa \\ Department of Applied Physics, Hokkaido University, Sapporo 060-8628, Japan
}

\begin{abstract}
Pulse compression of single 32.6-eV high harmonic pulses from a time-delay compensated monochromator was demonstrated down to $11 \pm 3$ fs by compensating the pulse front tilt. The photon flux was intensified up to $5.7 \times 10^{9}$ photons $/ \mathrm{s}$ on target by implementing high harmonic generation under a phase matching condition in a hollow fiber used for increasing the interaction length.
\end{abstract}

\section{Introduction}

High harmonics, which are generated under the nonlinear interaction between atoms and ultrashort laser pulses, can be compact table-top ultrashort light sources in the extreme ultraviolet (XUV) and soft x-ray regions. However, it is not straightforward to apply high harmonics to spectroscopy, because many multiple orders of high harmonics are generated collinearly with intense driving laser pulses. It is necessary to choose an appropriate harmonic order for application, especially for spectroscopy to gain insight into electronic structures of materials. To select a single harmonic with the pulse duration preserved, a time-delay compensated monochromator (TDCM), consisting of a pair of gratings to compensate for the pulse-front tilt shown in Fig. 1, was proposed and has been experimentally and theoretically investigated [1-4] In TDCM, the first grating and the slit separate the harmonic orders and then the second grating corrects the pulse-front tilt introduced by the first grating.

In this paper, we demonstrate the pulse compression of the selected high harmonics pulses from a TDCM and also the enhancement of the conversion efficiency by phase matching. Although we constructed a TDCM in the previous work and the pulse duration was compressed to $47 \mathrm{fs}$, there still remains a question that what limits the compressed pulse duration [4]. Especially, we are interested in the accuracy required for constructing the monochromator. Then, in this work, we compressed the $21 \mathrm{st}$ harmonic pulses $(=32.6 \mathrm{eV})$ down to 11 \pm 3 fs by adjusting the slit position located between the gratings. To improve the conversion efficiency, high harmonics were generated in a hollow fiber filled with rare gas to increase the

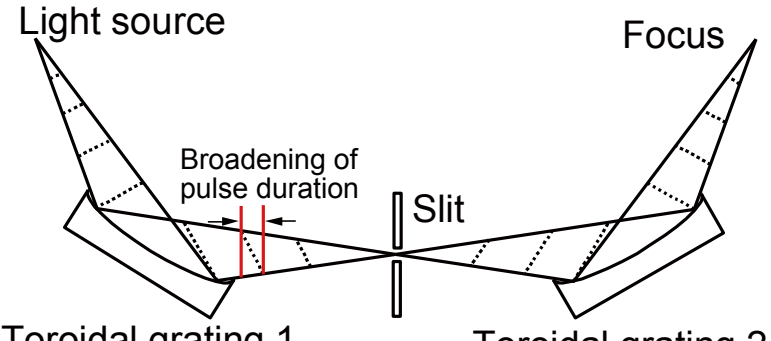

Toroidal grating 1

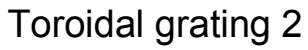

Fig. 1. Schematic of a time-delay compensated monochromator. The dotted lines indicate the pulse front inside the beam. The distance between the red lines corresponds to the broadening of the pulse duration. 
interaction length under a phase-matched condition, which was found by changing gas pressure inside the fiber [5].

\section{Experiment}

The laser system served for high harmonic generation was as follows: 0.7-mJ Ti:sapphire laser pulses with a pulse duration of $26 \mathrm{fs}$ at a repetition rate of $1 \mathrm{kHz}$ were focused into a 1-cm-long, 300$\mu \mathrm{m}$-diameter hollow fiber filled with static krypton gas by a concave mirror with $50-\mathrm{cm}$ radius. The peak intensity of the laser pulse inside the fiber was $4.7 \times 10^{13} \mathrm{~W} / \mathrm{cm}^{2}$. The position of the fiber was adjusted along the direction of the beam propagation so that the output power was maximized. The photon flux of the separated high harmonic was measured using by a calibrated XUV photodiode. The temporal duration of the selected high harmonic pulses was measured by observing the temporal evolution of the sideband peaks in photoelectron spectra of $\mathrm{Ne}$ that were caused by the two-photon free-to-free transition under simultaneous irradiation of both XUV and fundamental photons.

\section{Pulse compression of high harmonic pulses}

he difference in the optical path lengths of two diffracted beams from the adjacent grooves on a grating is a wavelength $\lambda$ in the case of the first order diffraction and the total difference in the optical path length across the beam amounts to $N \lambda$, where $N$ is the total number of illuminated grooves. Thus, although the wave front of the beam is still preserved after diffraction, the pulse front is tilted and the effective pulse duration is stretched by $N \lambda / c$. The basic idea of TDCM is to compensate for this optical path difference $N \lambda$ by placing the second grating at the symmetrical position with respect to the slit. For complete compensation, at least, the illuminated areas of both grating should be equal. Experimentally, the illuminated area can be changed with the slit position in the Fourier plane of the first grating between the toroidal gratings.

Thus, the pulse stretch of the 21 st harmonic in our TDCM system was estimated by a numerical simulation using the given parameters of the toroidal grating. The solid line in Fig. 2c shows the calculated pulse stretch arising from unequal irradiation areas on the gratings as a function of the slit position. At a slit position of $3 \mathrm{~mm}$, the irradiated areas of two gratings become equal and the pulse stretch is about $100 \mathrm{fs}$ with $5-\mathrm{mm}$ displacement of the slit. Therefore, it might be possible to compress the pulse duration by the shift of the slit position by several millimeters.

Therefore, we measured the pulse duration of the 21 st harmonic as a function of the slit position for
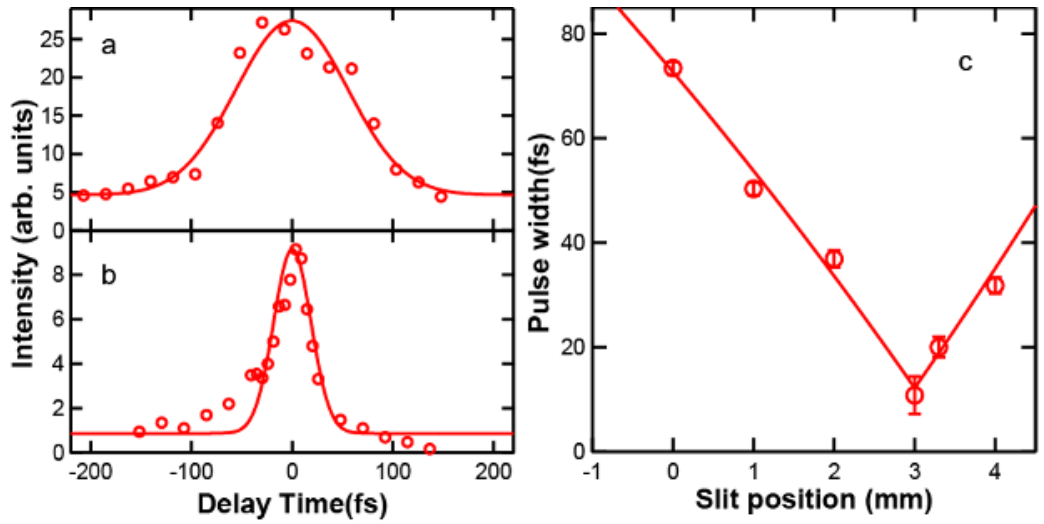

Fig. 2. Cross-correlation between the 21st harmonic and fundamental pulses at slit positions of (a) $0 \mathrm{~mm}$ and (b) $3 \mathrm{~mm}$. The solid lines are the results of fitting to Gaussian functions.

(c) Pulse duration of the 21st harmonic pulse as a function of slit position. The solid line is the numerically calculated pulse stretch shifted by the measured shortest pulse duration. 
pulse compression. Figures $2 \mathrm{a}$ and $2 \mathrm{~b}$ show the crosscorrelation between the 21 st harmonic and fundamental pulses at slit positions of 0 and of $3 \mathrm{~mm}$, respectively. Here, the origin of the slit position was defined at the slit position just after the initial experimental setup. The pulse duration of a selected harmonic pulse was obtained by deconvoluting a high harmonic pulse from the crosscorrelation by assuming harmonic and fundamental pulses as Gaussian functions. Figure 3c shows that the pulse duration was compressed down to $11 \pm 3 \mathrm{fs}$ at a slit position of $3 \mathrm{~mm}$ from the initial position. Further shift increased the pulse duration. The experimentally measured pulse duration was explained very well without free parameters. Therefore, the deviation of the incident angle from the optimum is the most crucial parameter to construct TDCM. To reduce the broadening within $10 \%$ from the optimum, the slit position should be determined within $0.1-\mathrm{mm}$ accuracy.

\section{Enhancement of the output flux by phase matching}

To find a phase-matched condition for high harmonic generation to increase the output power experimentally, the pressure dependence of the intensity of the 21 st harmonic, generated from $\mathrm{Kr}$ gas, was measured and is shown in Fig. 3. When the length of the hollow fiber was $2 \mathrm{~cm}$, the output intensity was found to have a maximum around 18 Torr, shown in Fig. 3a. On the other hand, when the interaction length was $1 \mathrm{~cm}$, the photon number increased with pressure and reached the intensity saturation at more than 90 Torr as shown in Fig. 3b. The maximum output numbers $5.7 \times 10^{9}$ photons/s on target was obtained when the $1-\mathrm{cm}$ hollow fiber filled with 120-Torr $\mathrm{Kr}$ gas. Furthermore, a photon flux of $7.1 \times 10^{9}$ photons/s was obtained at 19 th harmonic $(=29.6 \mathrm{eV})$. Numerical simulations shown by the solid lines predict that the negative refractive index induced by ionization is crucial for phase matching. The output photon flux from a TDCM beamline was comparable to that of a synchrotron radiation facility.
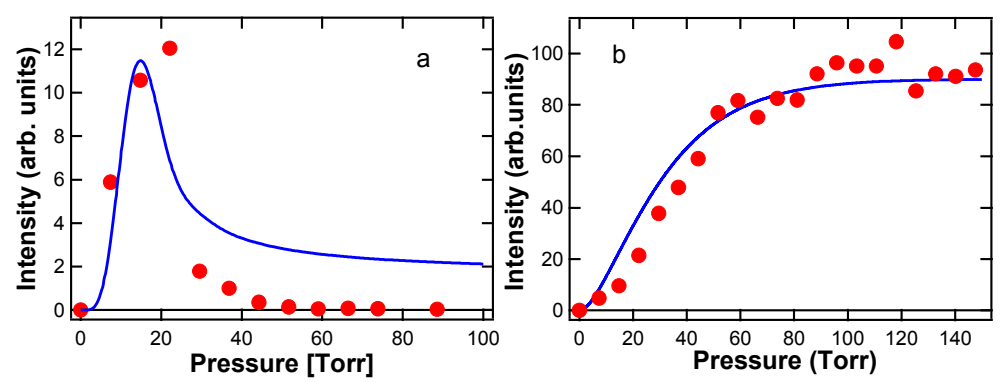

Fig. 3. Pressure dependence of the intensity of the 21 st harmonic at a fiber length of a) 2 and b) $1 \mathrm{~cm}$ shown by solid circles. The solid lines are the calculated phase matching factors.

\section{References}

1. P. Villoresi, Appl. Opt. 38, 6040 (1999).

2. L. Nugent-Glandorf, M. Scheer, D. A. Samuels, V. M. Bierbaum, and S. R. Leone, Rev. Sci. Instrum. 73, 1875 (2002).

3. L. Poletto, P. Villoresi, E. Benedetti, F. Ferrari, S. Stagira, G. Sansone, and M. Nisoli, Opt. Lett. 32, 2897 (2007).

4. M. Ito, Y. Kataoka, T. Okamoto, M. Yamashita, and T. Sekikawa, Opt. Express 18, 6071 (2010),

5. H. Igarashi, A. Makida, M. Ito, and T. Sekikawa, Opt. Express 20, 3725 (2012). 